\title{
The Theory of Vector-Valued Function in Locally Convex Space
}

\author{
Lixin Ma \\ Department of Mathematics, Dezhou University, Dezhou, China \\ Email: 735363648@qq.com
}

Received June 17, 2012; revised July 17, 2012; accepted July 24, 2012

\begin{abstract}
In this paper, the vector-valued regular functions are extended to the locally convex space. The residues theory of the functions in the locally convex space is achieved. Thereby the Cauchy theory and Cauchy integral formula are extended to the locally convex space.
\end{abstract}

Keywords: Locally Convex Space; Regular Function; Residue

\section{Introduction}

The properties of analytic functions have been given in references $[1,2]$. The theory of analytic functions was extended to vector valued function in reference [3].

In this paper, we extended the theory of vector valued function to locally convex space.

Let $E$ be a complete Hausdorff locally convex space on the real or complex domain $D$, and $P$ be the sufficient directed set of semi norms which generates the topology of $E$. We denote the ad joint space of $E$ by $E^{\prime}$, i.e. $E^{\prime}$ is the set of linear bounded functions on $E$.

Definition 1 Let $f(z)$ be a vector function defined on a domain $D$ with values in $E$. If there is an element $f^{\prime}(z) \in E$ such that the difference quotient

$\frac{f(z+h)-f(z)}{h}$ tends weakly(strongly) to $f^{\prime}(z)$ as $h \rightarrow 0$, we call $f^{\prime}(z)$ the weakly (strongly) derivative of $f(z)$ at $z$. We also say that $f(z)$ is weakly (strongly) derivative at $z$ in $D$. We call $f(z)$ weakly (strongly) derivative in $D$.

Definition 2 A vector function $f(z)$ is

1) weakly continuous at $z=z_{0}$ if

$\lim _{t \rightarrow t_{0}}\left|\varphi\left(f(z)-f\left(z_{0}\right)\right)\right|=0$ for each $\varphi \in E^{\prime}$.

2) strongly continuous at $z=z_{0}$ if

$\lim _{t \rightarrow t_{0}}\left|\varphi\left(f(z)-f\left(z_{0}\right)\right)\right|=0$ for each $\varphi \in E^{\prime}$.

Definition 3 A vector function $f(z)$ is said to be regular in $D$ if $\varphi(f(z))$ is regular for every $\varphi \in E^{\prime}$, where range of $f(z)$ is in $E$. If a vector valued function $f(z)$ is regular in $C$, then $f(z)$ is called an entire function or said to be entire.
Theorem 1 [4] (Cauchy) If $f(z)$ is a regular vector-valued function on the domain $D$ with values in the locally convex space $E$. Let $\gamma$ be a closed path in $D$, and assume that $\gamma$ is homologous to zero in $D$, then

$$
\int_{\gamma} f(z) \mathrm{d} z=0
$$

where $c$ is a circle.

Proof For any linear bounded functional $\varphi \in E^{\prime}$, we have

$$
\varphi\left(\int_{c} f(z) \mathrm{d} z\right)=\int_{c} \varphi(f(z)) \mathrm{d} z=0
$$

Hence

$$
\int_{c} f(z) \mathrm{d} z=0
$$

Theorem 2 [5] (Cauchy integral formula) Let $f(z)$ be a regular vector-valued function on the domain $D$ with values in the locally convex space $E$. Let $\gamma$ be a closed path in $D$, and assume that $\gamma$ is homologous to zero in $D$, and let $z$ be in $D$ and not on $\gamma$. Then

$$
\frac{1}{2 \pi i} \int_{\gamma} \frac{f(t)}{t-z} \mathrm{~d} t=n(\gamma, z) f(z)
$$

where $n(\gamma, z)$ is the index of the point $z$ with respect to the curve $\gamma$.

Proof For any linear bounded functional $\varphi \in E^{\prime}$, we have

$$
\begin{aligned}
\varphi\left(\frac{1}{2 \pi i} \int_{\gamma} \frac{f(z)}{t-z} \mathrm{~d} t\right) & =\frac{1}{2 \pi i} \int_{\gamma} \frac{\varphi(f(z))}{t-z} \mathrm{~d} t \\
& =n(\gamma, z) \varphi(f(z))
\end{aligned}
$$

Then 


$$
\frac{1}{2 \pi i} \int_{\gamma} \frac{f(t)}{t-z} \mathrm{~d} t=n(\gamma, z) f(z)
$$

\section{The Main Conclusions}

Theorem 3 Given the power series

$$
\sum_{n=0}^{\infty} a_{n}\left(z-z_{0}\right)^{n}, a_{n} \in E .
$$

Set $\frac{1}{\rho}=\lim _{n \rightarrow \infty} \sup \left(\rho\left(a_{n}\right)\right)^{\frac{1}{n}}$. Then the power series

is absolutely convergent for $\left|z-z_{0}\right|<\rho$ and divergent for $\left|z-z_{0}\right|>\rho$. The power series (2) convergence to a regular function on $\left|z-z_{0}\right|<\rho$ with values in $E$, the convergence being uniform in every circle of radius less than $\rho$.

Proof First, we will prove the power series (2) is absolutely convergent for $\left|z-z_{0}\right|<\rho$ and divergent for $\left|z-z_{0}\right|>\rho$.

By Theorem 1, for any $p \in P$, we have

$$
p\left(a_{n}\right) \leq \frac{1}{2 \pi} \int_{C} \frac{p(f(z))}{\left(z-z_{0}\right)^{n+1}} \mathrm{~d} z \leq M_{r} r_{-n},
$$

where $M_{r}=\max \left\{p(f(z)), z \in C, C:\left|z-z_{0}\right|=r<\rho\right\}$.

Let $r=\rho-\varepsilon$, then

$$
\begin{aligned}
p\left(\sum_{n=0}^{\infty} a_{n}\left(z-z_{0}\right)^{n}\right) & \leq \sum_{n=0}^{\infty} p\left(a_{n}\right)\left|z-z_{0}\right|^{n} \\
& \leq M_{\rho-\varepsilon} \sum_{n=0}^{\infty} \frac{\left|z-z_{0}\right|^{n}}{(\rho-\varepsilon)^{n}},
\end{aligned}
$$

where $\left|z-z_{0}\right|<\rho-\varepsilon$. Thus the power series (2) is absolutely convergence. But for $\left|z-z_{0}\right|>\rho$, if we suppose the power series (2) is convergence, it is contradict with the radius is $\rho$. So the power series (2) is absolutely convergent for $\left|z-z_{0}\right|<\rho$ and divergent for $\left|z-z_{0}\right|>\rho$.

Secondly, for any linear bounded functional $\varphi \in E^{\prime}$, we have

$$
\varphi(f(z))=\sum_{n=0}^{\infty} \varphi\left(a_{n}\right)\left(z-z_{0}\right)^{n},\left|z-z_{0}\right|<\rho .
$$

The right side series convergence to a regular function on $\left|z-z_{0}\right|>\rho$ with values in $E$. So $f(z)$ is regular in the circle and the convergence being uniform.

Definition 4 Let $f(z)$ have an isolated singularity at $z=z_{0}$ and let

$$
f(z)=\sum_{n=-\infty}^{\infty} a_{n}\left(z-z_{0}\right)^{n}
$$

where

$$
a_{n}=\frac{1}{2 \pi i} \int_{\gamma} \frac{f(z)}{z-z_{0}} \mathrm{~d} z
$$

be its Laurent Expansions about $z=z_{0}$. The residue of $f(z)$ at $z=z_{0}$ is the coefficient $a_{-1}$. Denote this by $\operatorname{Re} s f(a)$.

Theorem 4 Let $f(z)$ be a regular vector-valued function except for a finite number of points $z_{1}, z_{2}, \cdots, z_{k}$ in the domain $D$. Let $\gamma$ be a closed path in $D$, and assume that $\gamma$ is homologous to zero in $D$, and let $z$ be in $D$ and not on $\gamma$. Then

$$
\frac{1}{2 \pi i} \int_{\gamma} \frac{f(t)}{t-z} \mathrm{~d} t=\sum_{j=1}^{k} n\left(\gamma, z_{j}\right) \operatorname{Res}_{z=z_{j}} f(z)
$$

Proof For any linear bounded functional $\varphi \in E^{\prime}$, we have

$$
\varphi \int_{\gamma} f(z) \mathrm{d} z=\int_{\gamma} \varphi(f(z)) \mathrm{d} z=\sum_{j=1}^{k} n\left(\gamma, z_{j}\right) \operatorname{Res}_{z=z_{j}} \varphi(f(z)) .
$$

Then

$$
\frac{1}{2 \pi i} \int_{\gamma} \frac{f(t)}{t-z} \mathrm{~d} t=\sum_{j=1}^{k} n\left(\gamma, z_{j}\right) \operatorname{Res}_{z=z_{j}} f(z)
$$

\section{Theorem 5} then

1) If $f(z)$ has a pole of order one at a point $z_{0}$

$$
\operatorname{Re}_{z=a} f\left(z_{0}\right)=\lim _{z \rightarrow a}\left(z-z_{0}\right) f(z)
$$

2) If $f(z)$ has a pole of order $n$ at a point $z_{0}$ then

$$
\operatorname{Res}_{z=a} f\left(z_{0}\right)=\frac{1}{(n-1) !} \lim _{z \rightarrow a} \frac{\mathrm{d}^{n-1}}{\mathrm{dz}^{n-1}}\left(z-z_{0}\right)^{n} f(z)
$$

Proof Because $f(z)$ has a pole of order $n$ at a point $z_{0}$, then $f(z)$ can be written in the form

$$
f(z)=\frac{\phi(z)}{\left(z-z_{0}\right)^{n}}
$$

where $\phi(z)$ is regular and nonzero at $z_{0}$.

So $\phi(z)$ has a power series representation

$$
\phi(z)=\sum_{n=0}^{\infty} \frac{\phi^{(n)}\left(z_{0}\right)}{n !}\left(z-z_{0}\right)^{n}
$$

in some neighborhood of $z_{0}$. It follows that

$$
f(z)=\sum_{0}^{\infty} \frac{\frac{\phi^{(n)}\left(z_{0}\right)}{n !}\left(z-z_{0}\right)^{n}}{\left(z-z_{0}\right)^{m}}
$$

in some neighborhood of $z_{0}$. Then we have formula (7)

$$
\operatorname{Re}_{z=a} f\left(z_{0}\right)=\frac{1}{(n-1) !} \lim _{z \rightarrow a} \frac{\mathrm{d}^{n-1}}{\mathrm{~d} z^{n-1}}\left(z-z_{0}\right)^{n} f(z)
$$

Obviously, when $n=1$, the formula (7) is formula (6). Theorem 6 If 


$$
f(z)=a_{m}\left(z-z_{0}\right)^{m}+a_{m+1}\left(z-z_{0}\right)^{m+1}+\cdots,
$$

So

where $z_{k} \in E$ for $k=m \cdot m+1, \cdots$, and if $a_{m}^{-1}$ exists, then $f^{-1}(z)$ exist and has a pole with order $m$ at $z_{0}$.

\section{Proof Since}

$$
\frac{f(z)}{\left(z-z_{0}\right)^{m}}=a_{m}+a_{m+1}\left(z-z_{0}\right)+a_{m+2}\left(z-z_{0}\right)^{2}+\cdots .
$$

For any linear bounded functional $\varphi \in E^{\prime}$, we have

$$
\varphi\left(\frac{f(z)}{\left(z-z_{0}\right)^{m}}-a_{m}\right)<\varphi\left(a_{m}^{-1}\right)^{-1},
$$

as $0<\left|z-z_{0}\right|<\delta$,

where $\delta$ is sufficiently small. Thus

$$
\varphi\left(I-\frac{f(z)}{\left(z-z_{0}\right)^{m}} a_{m}^{-1}\right)=\varphi\left(\left(a_{m}-\frac{f(z)}{\left(z-z_{0}\right)^{m}}\right) a_{m}^{-1}\right)<1 .
$$

It follows that

$$
\left(\frac{f(z)}{\left(z-z_{0}\right)^{m}} a_{m}^{-1}\right)^{-1}=I+\sum_{n=1}^{\infty}\left(I-\frac{f(z)}{\left(z-z_{0}\right)^{m}} a_{m}^{-1}\right) .
$$

Therefore

$$
f^{-1}(z)=\frac{a_{m}^{-1}}{\left(z-z_{0}\right)^{m}}+\frac{b_{m+1}}{\left(z-z_{0}\right)^{m+1}}+\frac{b_{m+2}}{\left(z-z_{0}\right)^{m+2}}+\cdots,
$$

where $b_{m+1}, b_{m+2}, \cdots \in E$.

Remark: $a_{m}^{-1}$ exist, this condition is important.

For example, in $Z_{2}$, we define $x \cdot y=\left(x_{1} y_{1}, x_{2} y_{2}\right)$, where $x=\left(x_{1}, x_{2}\right), y=\left(y_{1}, y_{2}\right)$ and For any linear bounded functional $\varphi \in E^{\prime}$

$$
\varphi(x, y)=\max \left\{\left|x_{i} y_{i}\right|\right\} \leq \max \left\{\left|x_{i}\right|\right\} \max \left\{\left|y_{i}\right|\right\}=\varphi(x) \varphi(y) \text {. }
$$

Thus $Z_{2}$ is a $B$-algebra, and $x^{-1}=\left(\frac{1}{x_{1}}, \frac{1}{x_{2}}\right)$. We set

$$
f(z)=\left(z, z^{3}\right)=z_{1} e_{1}+z^{3} e_{2},
$$

where $e_{1}=(1,0)$ and $e_{2}=(0,1)$. It follows that $z=0$ is zero with order one, but

$$
f^{-1}(z)=\left(\frac{1}{z}, \frac{1}{z^{3}}\right)=\frac{1}{z} e_{1}+\frac{1}{z^{3}} e_{2}
$$

With order three.

Theorem 7 If $f(z)$ and $g(z)$ are regular in $D$ with values in $E$ and if $f\left(z_{n}\right)=g\left(z_{n}\right), n=1,2, \cdots$, the points $\left\{z_{n}\right\}$ having a limit point in $D$, then $f(z)=g(z)$ in $D$.

Proof For any linear bounded functional $\varphi \in E^{\prime}$, we have

$$
\varphi(f(z))=\varphi(g(z)), z \in D
$$

$$
f(z)=g(z), z \in D .
$$

Theorem 8 Let $f(z)$ be defined in a domain $D$ of the extended plane and on its boundary $C$, regular in $D$ and strongly continuous in $D \cup C$. If

$$
\{\sup \{p(f(z))\}: z \in C\}=M,
$$

then either $p(f(z))=M$ or $p(f(z))<M$ in $D$.

Proof For any linear bounded functional $\varphi \in E^{\prime}$, we have

$$
\varphi(f(z)) \leq M, z \in D \cup C .
$$

But except $\varphi(f(z))$ is constant, $\varphi(f(z))<M, z \in D$. So either $p(f(z))=M$ or $p(f(z))<M$ in $D$.

Remark: Unlike the classical case, $p(f(z))$ may have a minimum other zero in $D$ as the following example shows.

For example, Let $B$ be a Banach space of complex pairs, $z=\left(z_{1}, z_{2}\right)$, where $\|z\|=\left(\left|z_{1}\right|,\left|z_{2}\right|\right)$.

Set

$$
a_{1}=(1,0), a_{2}=(0,1),
$$

Then

$$
f(z)=a_{1}+a_{2} z,\|f(z)\|=1 \text { for }|z| \leq 1
$$

and

$$
\|f(z)\|=|z| \text { for }|z|>1 .
$$

Theorem 9 If $f(z)$ is regular in $D$, and if $p(f(z))$ is bounded in $D$, then $f(z) \equiv$ constant element.

Proof For any linear bounded functional $\varphi \in E^{\prime}$, we have

$$
\varphi(f(z)) \leq p(\varphi) p(f(z)) .
$$

So $\varphi(f(z))$ is bounded in $D$, then $\varphi(f(z))$ is constant.

Suppose $f(z)$ is not constant, then exist two point $z_{1}, z_{2}$ such that

$$
f\left(z_{1}\right)=f_{1}, f\left(z_{2}\right)=f_{2}, f_{1} \neq f_{2} .
$$

Thus exist $\varphi \in E^{\prime}$ satisfy

$$
\varphi_{0}\left(f\left(z_{1}\right)\right) \neq \varphi_{0}\left(f\left(z_{2}\right)\right) \text {. }
$$

This is contradict with $\varphi(f(z))$ is constant. So $f(z) \equiv$ constant element.

Theorem 10 If $f(z)$ is regular in the unit circle, satisfy the condition $p(f(z)) \leq M$ and $f(0)=0$. Then

$$
p(f(z)) \leq M|z|,|z|<1 .
$$

Proof For any linear bounded functional $\varphi \in E^{\prime}$, we 
have

$$
\varphi(f(z)) \leq p(\varphi) p(f(z)) \leq M p(f(z)),|z|<1 .
$$

Since every point $z_{0} \in E$, their exist a bounded function $\varphi$ such that

$$
\varphi\left(z_{0}\right)=p\left(z_{0}\right), p(\varphi)=1 .
$$

So

$$
\varphi(f(z))=p(f(z)), p(\varphi)=1 .
$$

Then

$$
p(f(z)) \leq M|z|,|z|<1
$$

\section{REFERENCES}

[1] L. V. Ahlfors, "Complex Analysis," McGraw-Hill, New
York, 2004.

[2] S. Lang, "Complex Analysis," Springer-Verlag, New York, 2003.

[3] C. G. Hu, "Vector-Valued Function and Its Applications," Kluwer Academic Publishers, Dordrecht, Boston, London, 1992.

[4] C. P. Xie and Y. S. Zhao, "Basic Boundary Value Problem for Complex Harmonic Functions," Beijing Math, Vol. 3, No. 1, 1997, pp. 20-35.

[5] Z. Zhen, "Schwarz's Problems for Some Complex Partial Differential Equations of Second Order," Beijing Math, Vol. 2, No. 1, 1996, pp. 131-137. 